\title{
Biclonal Immunoglobulin M Dysglobulinaemia: Evolving Aspects in a Case of Primary Sjögren's Syndrome ${ }^{1}$ )
}

\author{
Françoise Pontet ${ }^{1}$, Chantal Halimi ${ }^{2}$, Anne Brocard $^{3}$ and Thierry Delacour ${ }^{3}$ \\ 1 Service de Biochimie, Hôpital Lariboisière, Paris, France \\ 2 Service de Médecine II, Centre hospitalier de Senlis, Paris, France \\ ${ }^{3}$ Service Laboratoire, Centre hospitalier de Senlis, Paris, France
}

Summary: The observation of suggestive clinical symptoms in a patient suffering from a Gougerot-Sjögren syndrome led to a search for a cryoglobulin. Unusual physico-chemical features of this cryoglobulin were discovered, using standard electrophoresis, immunoelectrophoresis, immunofixation and electroimmunotransfer. The main unusual finding was that the cryoprecipitate was made up of a biclonal IgM $\kappa$ associated to polyclonal IgG. Therefore, we suggest that this new form of cryoglobulin be classified as a subtype IIb, thus distinguishing two subtypes in the usual classification.

\section{Introduction}

Cryoglobulins have been classified into three types according to the characteristics of the constituting Ig (1): type I corresponds to the presence of a single monoclonal Ig; type II is formed of a monoclonal Ig and polyclonal Ig, frequently a monoclonal rheumatoid IgM complexed with polyclonal IgG; type III, in which only polyclonal Ig are found, in low concentrations. The first two types are mostly encountered in Waldenström macroglobulinaemia, myeloma, other lymphoproliferative diseases and immune disorders such as the GougerotSjögren syndrome. The third type is mostly encountered in auto-immune or infectious diseases (2). Certain cases of type II and type III are considered by some authors as the so-called "mixed essential cryoglobulins", many of which are now associated with hepatitis $C$ virus infection and mostly contain IgG (either monoclonal, bi- or multiclonal). They have been largely described and reviewed in recent years $(3,4)$. In contrast, biclonal IgM evolving towards monoclonal IgM, associated to a primary Gougerot-Sjögren syndrome has not been yet described, to our knowledge.

\footnotetext{
1) Funding organisation:

Association pour le développement et l'étude des techniques biologiques et pharmaceutiques, équipe de recherche $n^{\circ} 22$.

$\left.{ }^{2}\right)$ SSA, SSB: an antigen A, B characterized by sera of certain patients with Sjögren syndrome (SS)

$\mathrm{Sm}$ : antigen (nuclear glycoprotein) called according to the initials of the patient where it occurred

RNP: ribonucleoprotein

Scl70: an antigen of $M_{\mathrm{r}}=70000$ identified by sera of certain patients with sclerodermia (Scl) Jo-1: an antigen which is found in polymyositis and dermatomyositis.

Reference: Lakomek et al.; this J. 1983, 21:621-32.
}

\section{Materials and Methods}

Case history

A 65-year-old woman with primary Sjören syndrome known for 10 years, and assessed by labial salivary glands biopsy, was admitted at the hospital at the end of December 1994 because of a white, soft and painless lower limb oedema, pitting and rising up to the lumbar region. Clinical examination showed a blood pressure at $21.3 / 12.0 \mathrm{kPa}$, a $7 \mathrm{~kg}$ weight increase (present weight is $52.5 \mathrm{~kg}$ ). Raynaud's phenomenon, xerostomia and a definite xerophthalmia were present. Further clinical examination was non-contributive.

Biological features revealed an incomplete nephrotic syndrome, with proteinuria $(4.45 \mathrm{~g} / 24 \mathrm{~h}$ ), hypoprotidaemia $(43 \mathrm{~g} / \mathrm{l})$ and hypoalbuminaemia $(20.8 \mathrm{~g} / \mathrm{l})$. Urinary cytobacteriological tests showed a microscopic haematuria; electrophoresis of urinary proteins showed a predominance of albumin $(77.8 \%)$. Renal function was normal. $\gamma$-Glutamyltransferase was increased to 5 times the reference value.

A mixed, type II cryoglobulinaemia was present, the composition of which will be detailed below.

Immunological tests yielded the following results: presence of antinuclear antibodies at the $1 / 200$ dilution, with an M1 speckled aspect (by indirect immunofluorescence technique on Hep. 2 cells), and an SSA specificity (55 Units); other antibodies against soluble nuclear antigen, e. g. SSB, Sm, RNP, ScI 70 and Jo- $1^{2}$ ) were absent. Anti-mitochondria antibodies were present at $1 / 100$, with an $M 2$ aspect. Hepatitis B surface (HBs) antigen was not detected, serologic tests for hepatitis $\mathrm{C}$ were negative. Hepatitis $\mathrm{C}$ virus was absent, as shown by polymerase chain reaction. Complete haematological investigations including osteomedullary biopsy did not reveal any sign of tumoural development.

Renal biopsy showed a cryoglobulin related damage. There were neither extra-capillary crescents, nor tubulo-interstitial consequences. Anti-IgG and anti-IgM antisera revealed granular, mostly parietal positive staining corresponding to the cryoglobulin type.

Corticosteroid therapy $(1 \mathrm{mg} / \mathrm{kg} \cdot \mathrm{d}$, or $50 \mathrm{mg} / \mathrm{d})$ was started, associated to saluretic furosemide (Lasilix ${ }^{\circledR}, 60 \mathrm{mg} / \mathrm{d}$ ) and anti-hypertensive nicardifine (Loxen $50^{\circledR}, 3$ tablets, $150 \mathrm{mg}$ per day).

Evolution was favourable with regression of oedema, weight loss of $10 \mathrm{~kg}$. After a 2 month corticosteroid therapy, proteinuria dropped to $1.5 \mathrm{~g} / 24 \mathrm{~h}$, total serum protein raised to $60 \mathrm{~g} / 1$, as well 
as serum albumin ( $34 \mathrm{~g} / \mathrm{l})$. Cryoglobulin was still present. Corticosteroid therapy was then progessively reduced until a total span of 5 months. At the end of this treatment, proteinuria was almost physiological $(0.15 \mathrm{~g} / 24 \mathrm{~h})$ and cryoglobulin had disappeared.

Four months after stopping corticosteroid therapy, a recurrence of the nephrotic syndrome was observed, with proteinuria $(1.75 \mathrm{~g} /$ $24 \mathrm{~h})$ and hypoalbuminaemia $(28 \mathrm{~g} / \mathrm{l})$. Cryoglobulin reappeared, with an apparently different content, which was analyzed as described below. Haematological tests still revealed no sign of lymphoma.

Corticosteroid therapy $(50 \mathrm{mg} / \mathrm{d})$ was reintroduced allowing a drop of the proteinuria to $0.70 \mathrm{~g} / 24 \mathrm{~h}$, after 3 months. An alternate corticosteroid therapy was then started for 2 years: $50 \mathrm{mg} / \mathrm{kg} \cdot \mathrm{d}$, one every other day. At the present time, cryoglobulin is persistent, but nephrotic syndrome has not reappeared.

\section{Sera}

Two specimens were collected, on 23 December 1994 and 15 September 1995 (at the time of nephrotic syndrome recurrence).

Blood was drawn in a dry sampling vial and kept at $37^{\circ} \mathrm{C}$ until complete clotting. Then serum was separated, still at $37^{\circ} \mathrm{C}$.

\section{Screening for monoclonal immunoglobulins}

Cellulose acetate electrophoresis was performed with whole sera at $37^{\circ} \mathrm{C}$ in order to look for monoclonal immunoglobulins. All material used were from Sebia (92130 Issy-les-Moulineaux, France).

\section{Cryoglobulin detection}

After adding sodium azide $(100 \mathrm{mg} / \mathrm{l})$, sera were stored at $4{ }^{\circ} \mathrm{C}$ for 24 hours, then visually examined for cryoprecipitate.

\section{Study of cryoprecipitates}

The cryoprecipitates were isolated and washed three times with $\mathrm{NaCl} 150 \mathrm{mmol} / 1$ at $4{ }^{\circ} \mathrm{C}$ according to the classical technique (1). They were strictly maintained at a temperature between 37 and $40^{\circ} \mathrm{C}$ until analysis. Purity of cryoprecipitates was checked by immunoelectrophoresis, using an antiserum against human serum total proteins (5)

Typing of the cryoglobulins was performed by immunoelectrophoresis (6), immunofixation (7) and electroimmunotransfer (8).

The serum sampled on 23 December 1994 yielded a cryoprecipitate, termed A; serum sampled on 15 September 1995 yielded cryoprecipitate $\mathrm{B}$.

\section{Results and Discussion}

The known Gougerot-Sjögren syndrome of this patient has been complicated with a cryoglobulinaemia after a 10 year evolution, as described in frequent cases (2), $20 \%$ to $40 \%$ of the cases according to some authors (12, 14). This cryoglobulinaemia has been responsible for membrano-proliferative glomerulonephritis, as the origin of the nephrotic syndrome.

The aetiologic investigations of this Gougerot-Sjögren syndrome associated to a type II cryoglobulinaemia included the search for a lymphoproliferative syndrome: clinical examination found no peripheral lymphadenopathy, nor did thoraco-abdominal computer tomography scan find any profound adenopathy. Bone marrow aspirate and biopsy evidenced no malignant tumoural in- filtrate. Collagen disease could not be evidenced; a viral $\mathrm{C}$ infection could have been at the origin of the cryoglobulinaemia, but hepatitis $\mathrm{C}$ serology and hepatitis $\mathrm{C}$ virus PCR tests were negative.

The hypothesis of a primary biliary cirrhosis has been raised in light of both the increase of $\gamma$-glutamyltransferase to 5 times the reference value and the presence of type M2 anti-mitochondria antibodies.

A hepatic biopsy was done but the normal aspect of the biliary ducts did not lead to this diagnostic.

As for therapy, a favourable evolution has been induced by corticoids, but its initial 5 months duration proved to be insufficient, with recurrence of nephrotic syndrome and cryoglobulinaemia 4 months after. A lengthened corticosteroid therapy for at least one year was then started again.

Although no monoclonal immunoglobulin was detected by electrophoresis, an abundant cryoprecipitate was found in both sera after $24 \mathrm{~h}$ at $4{ }^{\circ} \mathrm{C}$. The precipitation temperature was determined to be between 37 and $30^{\circ} \mathrm{C}$

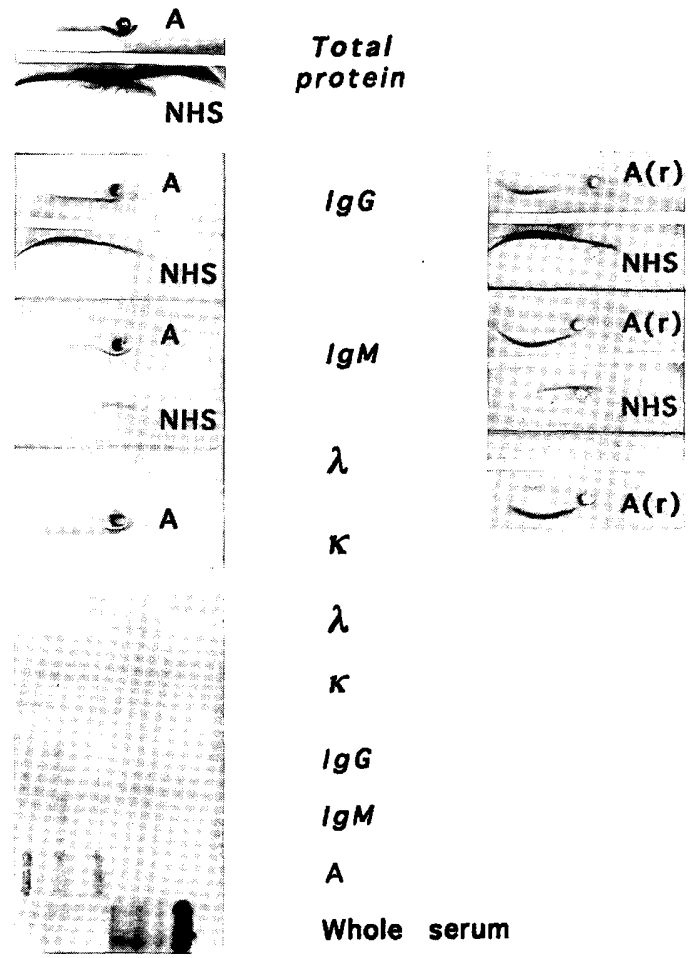

Fig. 1 Immunoelectrophoresis and immunofixation analysis of cryoprecipitate A (isolated from specimen drawn on 23/12/1994). Names of sera are in standard characters, names of antisera are in italics.

A: cryoprecipitate A

A(r): cryoprecipitate A reduced with cysteine hydrochloride

"NHS": normal human serum was used as control

Whole serum: matching whole serum

Total protein: anti-total protein antiserum

IgG: anti-IgG antiserum

IgM: anti-IgM antiserum

$\lambda$ : anti- $\lambda$ light chains (bound and free) antiserum

$\kappa$ : anti- $\kappa$ light chains (bound and free) antiserum 
in both cases, which is not very common (9). This case confirms that temperature is more important than quantity, as underlined by the same authors (9), because it explains typical clinical features despite the low concentration of this cryoglobulin. Immunoelectrophoresis (fig. 1 , anti-human serum total protein antiserum) demonstrated the absence of albumin, thus proving the purity of the cryoprecipitate. Anti-IgG, anti-IgM, anti-k light chains (bound and free) and anti- $\lambda$ light chains (bound and free) revealed the presence of a monoclonal IgMк and of polyclonal IgG. This located the cryoglobulin in type II of the current classification (1).

Immunoelectrophoresis of cryoprecipitate A exhibited a more abundant non-specific precipitate at the well cathodic edge than did that of the second precipitate. Consequently, immunofixation was performed to further explore these aspects.

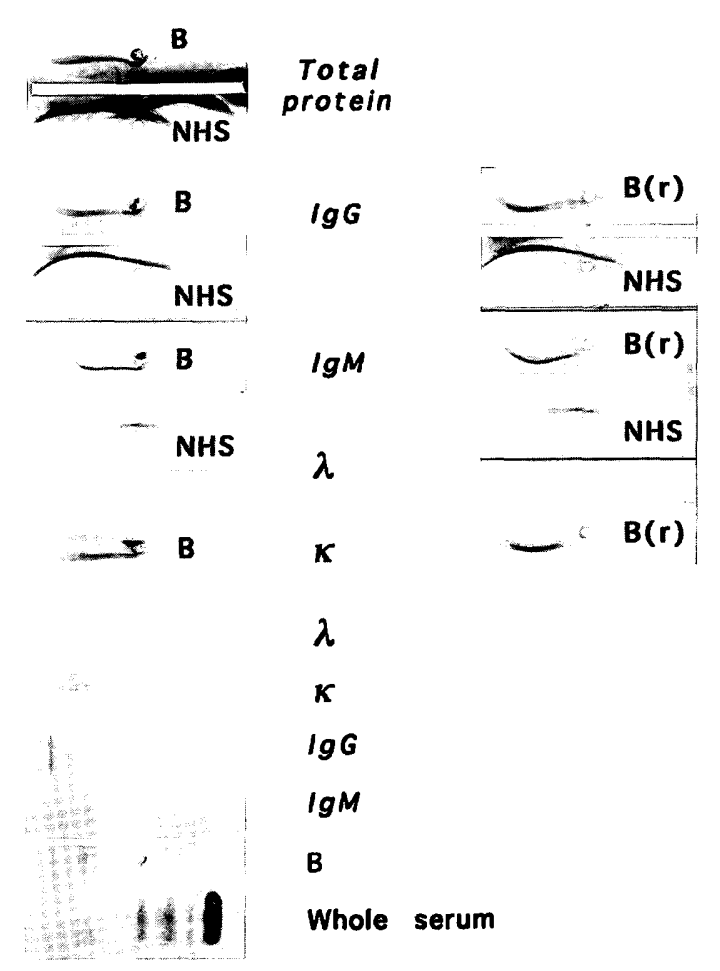

Fig. 2 Immunoelectrophoresis and immunofixation analysis of cryoprecipitate B (isolated from specimen drawn on 15/09/1995). Same legend as for figure 1 , with:

B: cryoprecipitate $B$

B(r): cryoprecipitate B reduced with cystein hydrochloride

Figure 2 shows that cryoprecipitate A contained two distinct bands reacting with anti- $\mu$ and anti- $\kappa$ antisera, while cryoprecipitate $B$ contained only one.

Electroimmunotransfer (8) was then performed to exclude the improbable presence of IgM monomers. As a matter of fact, IgM monomers are considered to lack the capacity to cryoprecipitate $(10,11)$. The results of electroimmunotransfer applied to cryoprecipitate A evidenced (fig. 3) that this cryoglobulin did not contain

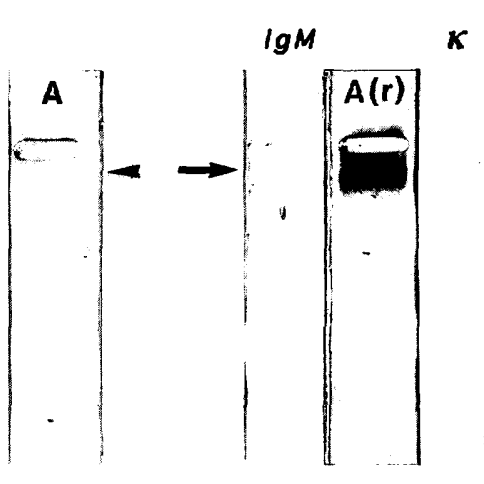

Fig. 3 Electroimmunotransfer of cryoprecipitate A (left), with control (right). Same legend as for figure 1.

- shows absence of monomers in cryoprecipitate A.

$\rightarrow$ shows control of electrophoresis -A(r)- and transfer -IgM, $\kappa$ - of reduced cryoprecipitate.

IgM monomers. Therefore, we were led to conclude that both monoclonal IgM were polymers.

Cryoprecipitate A is composed of two monoclonal common polymeric IgMK, or biclonal IgM (according to usual techniques), and polyclonal IgG, an observation that has never been described before to our knowledge. The closest resembling case was cited by Musset et al. and corresponded in fact to a pentaclonal Ig (1 IgG $+4 \mathrm{IgM})$, which was detectable in the corresponding whole serum. Thus, we propose subdividing type II of the current cryoglobulin classification into:

- type IIa, which would include cryoglobulins formed of one monoclonal Ig and polyclonal Ig (corresponding to type II of the previous classification);

- type IIb, which would be defined as cryoglobulins made up of two (or more) monoclonal Ig (multiclonal Ig) and polyclonal Ig. As suggested by previous authors (3), and by a frequent observation done in current practice, a progressive spreading of the cases could be distinguished inside this subtype, from bi- to tri- to multiclonal Ig, the latter could be the closest category to type III, whatever the concentrations found.

In our present case, cryoglobulin isolated from the first sample belongs to type IIb, cryoglobulin from the second sample is of type IIa.

The evolution within 9 months of a biclonal component of a cryoglobulin towards a monoclonal component seems interesting. Although it has been suggested (12) that oligoclonal lymphocyte expansion could precede a malignant state, there are no significant works, to our knowledge, stating that a change from circulating bi- or triclonal Ig to monoclonal Ig is a meaningful prognostic criterion. However, it has to be noted that, in our present case, the reappearance of a monoclonal cryoglobulin is correlative to the recurrence of the nephrotic syndrome, while it was initially biclonal, and had disappeared with corticosteroid therapy. The absence of any lymphoproliferative syndrome proves that this evolution does not 
correspond to a malignant dysglobulinaemia. It would rather make it classify as an "associated dysglobulinaemia", according to the authors' classification (5): "associated dysglobulinaemia" include all cases which are associated to an immunological disorder. This is consistent with the statement according to which lymphoma is a rare complication of primary Sjögren's syndrome (13).

As for the theoretical aspects of the cryoprecipitation phenomenon, our observation would tend to prove that in this case, the different Ig are not bound together. As a matter of fact, our results clearly show (even more evidently than in usual cases of type II cryoglobulins) that the two monoclonal IgM and the polyclonal IgG migrate independently: the different length and curve of the two IgG and IgM arcs imply two distinct fractions; the obvious difference in aspect and migration position of the immunofixation bands also suggest non-complexed types of molecules. Thus, they do not seem to be bound in any manner. Otherwise, we would get a similar aspect of the IgM and the IgG fractions, probably as a mixture or indefinite aspect in terms of arc length and curve in immunoelectrophoresis, and in the narrowness or broadness of the immunofixation bands.

So, as a recent work tends to show (14), we think that the Ig components of mixed (type II) cryoglobulins coprecipitate independently, without any bonding. Thus,

\section{References}

1. Brouet JC, Clauvel JP, Danon F, Klein M, Seligmann M. Biologic and clinical significance of cryoglobulins: a report of 86 cases. Am J Med 1974; 57:775-88.

2. Clauvel JP. Cryoglobulinémies. Rev Prat 1993; 43(3):302-5.

3. Musset L, Duarte F, Gaillard O, Thi Huong Du L, Bilala J, Galli J, et al. Immunochemical characterization of monoclonal IgG containing mixed cryoglobulins. Clin Immunol Immunopathol $1994 ; 70 ; 2: 166-70$.

4. Ronco PM, Mougenot B, Touchard G, Preud'homme JL, Aucouturier P. Renal involvement in hematological disorders: monoclonal immunoglobulins and nephropathy. Current Opinion in Nephrology and Hypertension 1995; 4:130-8.

5. Pontet F, Delauche-Cavalier MC. L'immunoglobuline monoclonale, marqueur tumoral du myélome et de la maladie de Waldenström. In: Girard ML, Rousselet F, Legrand A, editors. Aspects biologiques de la maladie cancéreuse. XXVIIe dimanches biologiques de Lariboisière; Apdila 1987.

6. Scheidegger JJ. Une microméthode de l'immunoélectrophorèse. Int Arch Allergy Appl Immunol 1955; 7:103-10.

7. Pontet F, Rousselet F. Application de l'immunofixation sur acétate de cellulose pour la classification rapide des immunoglobulinopathies biclonales. Ann Biol Clin 1982; 40:37-43.

8. Pontet F, Rousselet F. Monoclonal 75 IgM: a rapid screening technique. Clin Chim Acta 1984; 141:85-91.

9. Letendre L, Kyle RA. Monoclonal cryoglobulinemia with high thermal insolubility. Mayo Clin Proc 1982; 57:629-33.

10. Middaugh CR, Gerber-Jenson B, Hurvitz A, Paluszek A, Scheffel C, Litman GW. Physicochemical characterization of the reason for the cryoprecipitation phenomenon must be searched for in the structure of each molecule implied. The rather significant volume of the cryoprecipitate compared to the extremely low concentration of Ig (undetectable by electrophoresis) would suggest the presence of a non-protein substance. The type of binding involved in the cryoprecipitate, either of the antigenantibody type or resulting from another mechanism could not be explored on these specimens.

\section{Conclusion}

A new evolutive aspect of a cryoglobulinaemia is described in a patient with Gougerot-Sjögren syndrome. The serum drawn at first exhibits a peculiar type II cryoglobulin, made of a biclonal IgMк and of polyclonal IgG. Nine months later, the biclonal IgMk has evolved to a monoclonal IgMK, raising the question of the prognostic meaning of such observations.

It is suggested that the classification of cryoglobulins be modified, dividing type II into type IIa for monoclonal Ig and type IIb for bi or multiclonal Ig.

This work also shows that cryoprecipitation is not necessarily an antigen-antibody phenomenon and that its mechanism more probably proceeds from a structural basis of some Ig.

six monoclonal cryoimmunoglobulins: possible basis for colddependent insolubility. Proc Natl Acad Sci USA 1978; 75:3440-4.

11. Weber RJ, Clem LW. The molecular mechanism of cryoprecipitation and cold agglutination of an IgM lambda Waldenström macroglobulin with anti-Gd specificity: sedimentation analysis and localization of interacting sites. J Immunol 1981; 127 (1):300-15.

12. Oxholm P. Primary Sjögren syndrome - clinical and laboratory markers of disease activity. Semin Arthritis Rheum 1992; 22 (2):114-26.

13. De Bandt $M$. Rôle du virus de l'hépatite $C$ dans les cryoglobulinémies mixtes "essentielles» et le syndrome de GougerotSjögren. La Presse Médicale, 1992; 21 (37):1750-2.

14. Miescher PA, Huang Y-P, Izui S. Type II cryoglobulinemia. Semin Hematol 1995; 32 (1):80-5.

15. Panka DJ, Salant DJ, Jacobson BA, Minto AW, MarshakRothstein A. The effect of VH residues 6 and 23 on IgG3 cryoprecipitation and glomerular deposition. Eur J Immunol 1995; 25:279-84

\section{Received September 16, 1996/January 20, 1997}

Corresponding author: Françoise Pontet, Service de Biochimie, Hôpital Lariboisière, 2, rue Ambroise Paré, F-75010 Paris, France 